\title{
Sorção do herbicida imazaquin em Latossolo sob plantio direto e convencional
}

\author{
Maurílio Fernandes de Oliveira ${ }^{(1)}$, Ignácio Colonna ${ }^{(2)}$, Hélio Teixeira Prates(3), Evandro Chartuni Mantovani(3), \\ Reinaldo Lúcio Gomide ${ }^{(3)}$ e Rubem Silvério de Oliveira Júnior ${ }^{(4)}$
}

\begin{abstract}
(1)Purdue University, Dep. of Agronomy, 915 W. State Street, West Lafayette, IN 47907, USA. E-mail: moliveira@purdue.edu (2)University of Illinois Urbana-Champaign, Dep. of Crop Sciences, 1102 S. Goodwin Ave. Urbana, IL 61801, USA. E-mail: iacolonn@uiuc.edu (3)Embrapa Milho e Sorgo, Caixa Postal 151, CEP 35701-970 Sete Lagoas, MG. E-mail: htprates@cnpms.embrapa.br, evandro@cnpms.embrapa.br, gomide@cnpms.embrapa.br (4)Universidade Estadual de Maringá, Dep. de Agronomia, Av. Colombo 5790, CEP 87020-900 Maringá, PR. E-mail: rsojúnior@uem.br
\end{abstract}

Resumo - O objetivo deste trabalho foi avaliar o efeito da variabilidade do $\mathrm{pH}$ e da matéria orgânica de um Latossolo Vermelho distrófico, textura argilosa, sob diferentes manejos na retenção do herbicida imazaquin. A retenção deste herbicida foi medida pelo coeficiente de partição solo-água $\left(\mathrm{K}_{\mathrm{d}}\right)$ em amostras de solos coletados na camada superficial $(0-15 \mathrm{~cm})$ em área de 38 ha, sob irrigação com pivô central e plantio direto e convencional. A retenção do imazaquin foi maior nas áreas com menores valores de $\mathrm{pH}$ e altos teores de matéria orgânica, ou seja, nas áreas onde o plantio direto foi utilizado por longo tempo. $\mathrm{O}$ pH afetou a retenção do imazaquin por controlar tanto a natureza iônica dos componentes do solo, matéria orgânica e minerais de argila, quanto a sua especiação. $\mathrm{O}$ teor de argila não apresentou variação na área estudada, ou seja, não afetou a retenção do imazaquin. $\mathrm{O}$ modelo de predição da sorção do imazaquin no solo pela análise de regressão multivariada com duas variáveis independentes (teor de matéria orgânica e $\mathrm{pH})$ apresentou boa correlação $\left(\mathrm{R}^{2}=0,91\right)$.

Termos para indexação: imidazolinona, agricultura de precisão, matéria orgânica, pH, plantio convencional.

\section{Imazaquin herbicide sorption by an Oxisol with till and no-till management}

\begin{abstract}
The objective of this study was to evaluate the effect of spatial variation of $\mathrm{pH}$ and organic matter of a Dark Red soil, under different managements, on imazaquin herbicide sorption. Soil-water sorption partitioning coefficient $\left(\mathrm{K}_{\mathrm{d}}\right)$ was used in surface soil samples collected at $0-15 \mathrm{~cm}$ layer in a 38 ha area, under central pivot irrigation and with till and no-till management. Herbicide sorption was most strongly related to soil organic matter content and $\mathrm{pH}$, revealing greatest sorption in lower $\mathrm{pH}$ values with greater soil organic matter content. These samples were collected at no-till management area. The $\mathrm{pH}$ effects on imazaquin retention occur either by controlling the organic matter ionization form as also its speciation. The clay content in the whole area had no variation, however, it didn't affect the $\mathrm{K}_{\mathrm{d}}$ values. Using multivariate regression and two independent variables (soil organic matter content and $\mathrm{pH}$ ), the prediction of herbicide sorption by soil was good $\left(\mathrm{R}^{2}=0.91\right)$.

Index terms: imidazolinone, site-specific management, organic matter, $\mathrm{pH}$, conventional-tillage.
\end{abstract}

\section{Introdução}

A retenção é um dos processos mais importantes na determinação do destino de pesticidas no ambiente, afetando especialmente a disponibilidade para as plantas e a lixiviação para águas subterrâneas (Koskinen \& Harper, 1990). Um dos parâmetros comumente utilizados para quantificar a retenção de um pesticida pelo solo é o coeficiente de distribuição solo-água $\left(\mathrm{K}_{\mathrm{d}}\right)$. Altos e baixos valores desse coeficiente são indicativos de maior e menor retenção do pesticida pelo solo, respectivamente.

A retenção de um pesticida depende das propriedades físicas e químicas do solo e das moléculas dos pesticidas. Entre as propriedades do solo que se correlacionam com a retenção de um pesticida, a matéria orgânica é a que mais afeta a retenção de pesticidas não-polares (Koskinen \& Harper, 1990). Por sua vez, o conteúdo de argila e o valor de $\mathrm{pH}$ do solo têm-se mostrado importantes fatores em relação à sorção de pesticidas polares ou ionizáveis (Green \& Karickhoff, 1990).

O imazaquin pertence ao grupo químico das imidazolinonas e é registrado no Brasil para controle de plantas daninhas dicotiledôneas e algumas monocotiledôneas na cultura da soja, em aplicações na pré-emergência dessas espécies. Esse herbicida é considerado molécula anfótera, possuindo um ácido 
carboxílico e uma piridina como grupos funcionais (Mangels, 1991). Segundo esse autor, o imazaquin possui propriedades de ácido fraco, com $\mathrm{pK}_{\mathrm{a}}$ de 3,8. Em níveis elevados de $\mathrm{pH}$, a sorção do imazaquin é reduzida devido ao predomínio de sua forma aniônica. À medida que o valor do $\mathrm{pH}$ se aproxima e se iguala ao do $\mathrm{pK}_{\mathrm{a}}$, a concentração da forma não-ionizada (molecular) de herbicidas ácidos passa a ser igual à da forma aniônica predominante, e a molécula também pode exibir uma ligação com o solo típica de herbicidas não-iônicos. Em $\mathrm{pH}$ baixo e inferior ao $\mathrm{pK}_{\mathrm{a}}$, o imazaquin pode ser protonado a um cátion, que é fortemente sorvido por meio de troca de cátions (Mangels, 1991).

O comportamento das imidazolinonas no solo é fortemente afetado pelo pH (Loux \& Reese, 1992; Oliveira, 1998), pelos teores de carbono orgânico (Goetz et al., 1986; Che et al., 1992; Oliveira, 1998), pela textura (Loux et al., 1989), mineralogia (Che et al., 1992; Regitano et al., 1997; Oliveira, 1998; Ferreira et al., 2002) e retenção de água pelo solo (Goetz et al., 1986). A mobilidade e a eficiência geralmente decrescem com o aumento da sorção pelos colóides do solo.

A variabilidade das propriedades físicas e químicas do solo pode provocar uma retenção diferencial dos herbicidas que, por sua vez, reflete numa disponibilidade diferencial do herbicida na solução do solo, podendo gerar uma variação no controle das plantas daninhas (Gerstl, 2000), especialmente em grandes áreas cultivadas onde a aplicação do herbicida é feita numa única dose. A retenção diferencial também pode acarretar numa variabilidade no potencial de lixiviação do produto (Oliveira Junior et al., 1999).

A matéria orgânica foi descrita como o principal componente do solo que afeta a retenção diferencial de herbicidas (Blumhorst et al., 1990; Gonese \& Weber, 1998). Segundo esses autores, em herbicidas com valores de $\mathrm{K}_{\mathrm{d}}$ entre 1 e $10 \mathrm{~mL} \mathrm{~g}^{-1}$, pequenas mudanças na sorção podem acarretar grandes variações na quantidade do produto na solução do solo e, conseqüentemente, na sua lixiviação.

O objetivo desse trabalho foi avaliar o efeito da variabilidade do $\mathrm{pH}$ e da matéria orgânica de amostras coletadas num Latossolo Vermelho distrófico, textura argilosa, sob diferentes tipos de manejo na retenção do imazaquin.

\section{Material e Métodos}

O estudo da variabilidade da sorção do imazaquin no campo foi realizado numa área de 38 ha, sob irrigação com pivô central. Parte da área vem sendo utilizada sob sistema de plantio direto e, a outra, sob plantio convencional. A área da malha foi de 700 x $700 \mathrm{~m}$ localizada no centro do pivô, com $348 \mathrm{~m}$ de raio. Na amostragem, utilizou-se malha traçada na direção norte-sul/leste-oeste com 29 linhas em cada direção, cada linha com 29 pontos, sendo os pontos de amostragem georreferenciados a $25 \mathrm{~m}$. Foram coletadas 731 amostras de solo $(0-15 \mathrm{~cm})$, para análise das propriedades físico-químicas. A seguir, mapas foram processados, interpolando amostras na faixa de 0,5 unidades de $\mathrm{pH}$ e 0,7 unidades de matéria orgânica (MO), ambos utilizando grade de $25 \mathrm{~m}$. Duas variáveis foram selecionadas previamente após a análise estatística univariada. Selecionaram-se 12 amostras com valores de $\mathrm{pH}$ entre 5,0 e 5,5 e 5,5 e 6,0 dentro das faixas de $\mathrm{MO}<3,5$ e $>3,5$. A amostragem foi realizada de forma a obter o máximo de representatividade da área, tanto na parte sob manejo convencional quanto no plantio direto, com o menor número possível de amostras.

As 12 amostras selecionadas foram utilizadas no estudo de retenção do imazaquin no solo, ou seja, obtenção dos valores de $\mathrm{K}_{\mathrm{d}}$ (coeficiente de partição solo água). Para isso, utilizou-se o método de equilíbrio em partição. Os valores de $\mathrm{K}_{\mathrm{d}}$ foram calculados pela relação solo:solução 1:2, preliminarmente determinada. Desta forma, amostras de 2,5 $\mathrm{g}$ de solo foram colocadas em tubos de centrífuga, em que foram adicionados $5 \mathrm{~mL}$ da solução $0,01 \mathrm{M} \mathrm{de} \mathrm{CaCl}_{2}$ contendo o herbicida imazaquin (99,6\% de pureza) na concentração de 2,0 $\mathrm{mg} \mathrm{L}^{-1}$. Após agitação da mistura por 24 horas, os tubos foram centrifugados a $4.000 \mathrm{rpm}$ por 10 minutos. $\mathrm{O}$ sobrenadante foi recolhido, passado em filtro e membrana de náilon $0,2 \mu \mathrm{m}$. A quantificação do imazaquin foi feita em cromatógrafo líquido de alta eficiência (HPLC), marca Shimadzu, modelo LC 10A, detector UV/Vis. Foi utilizada a técnica do padrão externo, com curva de calibração linear baseada na integração da área do pico construída com padrão analítico.

A fase móvel consistiu da mistura água:acetonitrila (1:1) a pH 3,0, ajustado com ácido ortofosfórico 0,04M. Utilizou-se coluna Adsorbosil C-18, marca Alltech, com $4,6 \mathrm{~mm} \times 15 \mathrm{~cm}$, e diâmetro de poro de $5 \mu \mathrm{m}$. A cromatografia foi realizada à temperatura ambiente, com fluxo de $1,0 \mathrm{~mL} \mathrm{~min}^{-1}$ e detector UV com comprimento de onda a $240 \mathrm{~nm}$.

A concentração sorvida ( $\mathrm{mg} \mathrm{kg}^{-1}$ de solo) foi calculada pela diferença entre a concentração da solução inicial ou adicionada $\left(\mathrm{mg} \mathrm{L}^{-1}\right)$ e a concentração de equilíbrio 
(mg L-1). Os valores de $\mathrm{K}_{\mathrm{d}}\left(\mathrm{L} \mathrm{kg}^{-1}\right)$ foram obtidos dividindo-se a concentração sorvida pela concentração em equilíbrio.

Os valores de $\mathrm{pH}$ e os teores de matéria orgânica do solo das 12 amostras selecionadas foram analisados por meio da correlação de Pearson com os valores de $\mathrm{K}_{\mathrm{d}}$, utilizando-se procedimento de regressão (PROC REG) do sistema SAS. Em seguida, gerou-se uma equação de regressão linear múltipla para o coeficiente $\mathrm{K}_{\mathrm{d}}$, com os 12 valores de $\mathrm{pH}$ e matéria orgânica. Em virtude da baixa correlação entre o pH e MO $(r=0,09)$, utilizou-se modelo de regressão múltipla com ambas as variáveis preditivas de $K_{d}$. Essa equação foi utilizada com a finalidade de gerar valores de $K_{d}$ para a população de 647 amostras coletadas na área.

Em seguida, foram gerados mapas a fim de descrever a distribuição espacial de $\mathrm{K}_{\mathrm{d}}$, utilizando-se técnicas geoestatísticas baseadas em 647 valores de $\mathrm{K}_{\mathrm{d}}$ estimados. Semivariogramas foram obtidos, antes dos mapas serem gerados, visando informar sobre a estimação geoestatística do método de krigagem. Estes métodos foram descritos por Isaaks \& Srivastava (1990) e Goovaerts (1997). Com os novos valores de $\mathrm{K}_{\mathrm{d}}$ obtidos pela equação, variogramas de 35 e 125 graus foram gerados a fim de detectar a presença de anisotropia e o mapa da variabilidade espacial da sorção do imazaquin, utilizando-se processo de krigagem ordinário. Todas as análises geoestatísticas foram realizadas pelo programa GSLIB (Deutsch \& Journel, 1997), e o mapa final gerado pelo ArcView 3.2 com formato "raster" e célula final de tamanho de $10 \mathrm{~m}$.

\section{Resultados e Discussão}

A área estudada possui gradiente constante de elevação com os pontos mais altos na parte noroeste e os mais baixos ao sudeste do pivô. Estes pontos estão separados por $730 \mathrm{~m}$, possuindo diferença na elevação de $60 \mathrm{~m}$ (Figura 1). As 12 amostras utilizadas no cálculo dos coeficientes de sorção foram selecionadas de forma a abranger tanto áreas onde o plantio direto foi utilizado por longo tempo (região noroeste) quanto o convencional (sudeste). $\mathrm{Na}$ área de 38 ha, $69,5 \%$ possui teor de $\mathrm{MO}<3,5 \%$ (Figura 2), sendo que os maiores teores foram registrados na área em que se utilizou o plantio direto. A maioria das amostras possui valor de pH entre 5,0 e 6,0, com considerável variação dentro dessa faixa.
Os valores de pH e matéria orgânica das 12 amostras selecionadas para obtenção dos coeficientes de sorção $\left(\mathrm{K}_{\mathrm{d}}\right)$ estão próximos dos valores obtidos na população de amostras, o que indica que as amostras selecionadas apresentam boa representatividade da área estudada (Tabela 1). O teor de argila não apresentou

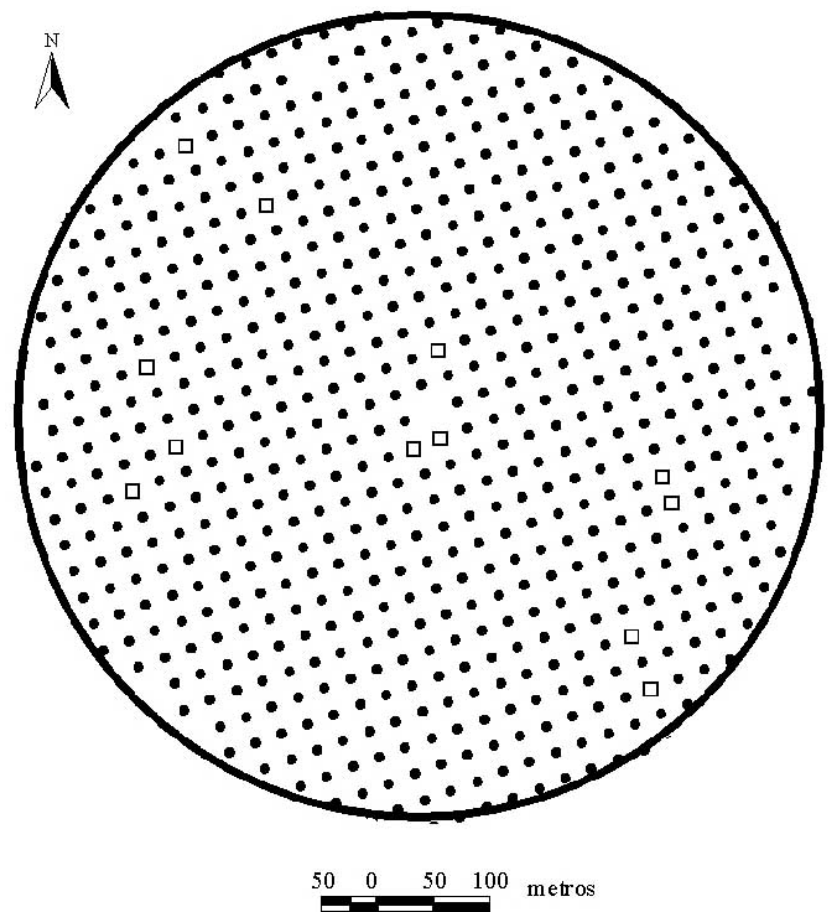

Figura 1. Esquema dos pontos selecionados $(\square)$ para obtenção dos valores de $\mathrm{K}_{\mathrm{d}}$ no total de pontos amostrados $(\bullet)$ na área do pivô central.

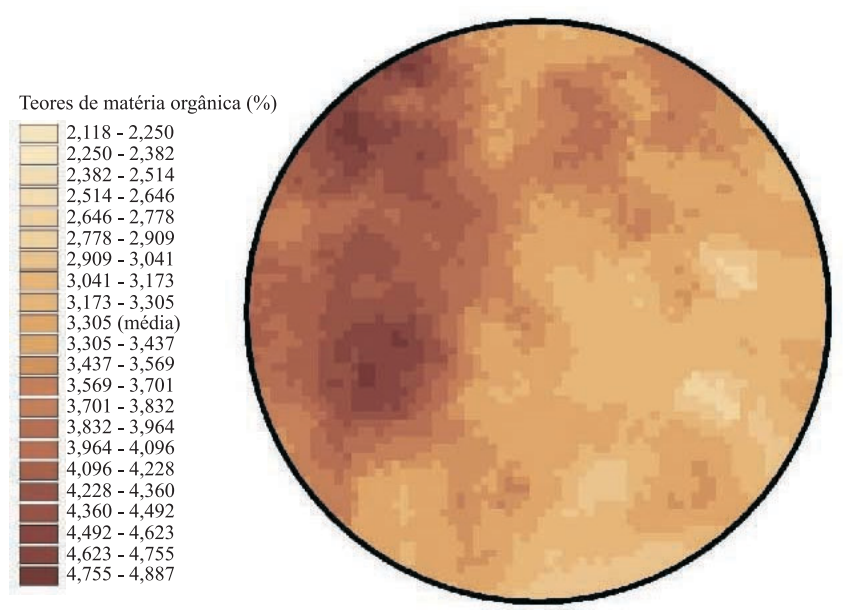

Figura 2. Teores de matéria orgânica do solo (\%) das amostras coletadas na área do pivô central. 
variação na área estudada (60\%), portanto, sem influência nos valores de $\mathrm{K}_{\mathrm{d}}$.

Os maiores valores de $\mathrm{K}_{\mathrm{d}}$ foram registrados nas amostras de menores valores de $\mathrm{pH}$, sendo a correlação entre esses parâmetros negativa a $5 \%$ de probabilidade $(r=-0,67)$. Loux et al. (1989), Stourgaard et al. (1990), Regitano et al. (1997, 2001) e Oliveira (1998) observaram decréscimo na retenção do imazaquin com o aumento do $\mathrm{pH}$ da solução do solo. Em valores mais elevados de $\mathrm{pH}$, as moléculas do imazaquin encontram-se predominantemente na forma ionizada, ou seja, com carga residual negativa, o que faz com que elas permaneçam na solução do solo e, portanto, com maior risco de lixiviação.

A correlação entre a sorção do imazaquin e o teor de MO foi positiva e significativa $(r=0,82)$ a $5 \%$ de probabilidade. Este alto valor de correlação pode ser explicado pelo fato de que a grande maioria das amostras com altos teores de MO possui baixos valores de $\mathrm{pH}$. Em baixos valores de $\mathrm{pH}$, tanto os grupos ionizáveis da $\mathrm{MO}$

Tabela 1. Valores de média, mediana, máxima, mínima, desviopadrão e coeficiente de variação para a população de amostras e para as 12 amostras de solo selecionadas.

\begin{tabular}{|c|c|c|c|c|}
\hline \multirow[t]{2}{*}{ Parâmetro } & \multicolumn{2}{|r|}{ População } & \multicolumn{2}{|r|}{ Amostras } \\
\hline & $\mathrm{pH}$ & Matéria orgânica & $\mathrm{pH}$ & Matéria orgânica \\
\hline Média & 5,6 & 3,3 & 5,5 & 3,6 \\
\hline Mediana & 5,6 & 3,2 & 5,6 & 3,5 \\
\hline Máxima & 7,7 & 6,4 & 6,0 & 5,4 \\
\hline Mínima & 4,4 & 0,1 & 5,1 & 2,6 \\
\hline Desvio-padrão & 0,35 & 0,68 & 0,36 & 0,79 \\
\hline Coef. de variação & $6,3 \%$ & $20,6 \%$ & $6,4 \%$ & $21,8 \%$ \\
\hline
\end{tabular}

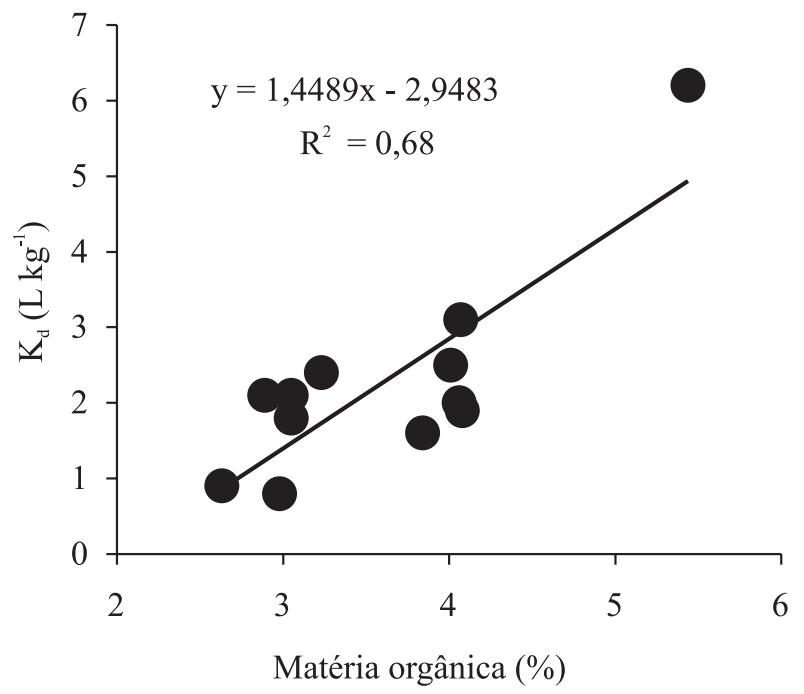

$\left(\mathrm{pK}_{\mathrm{a}} \sim 5,2\right)$ quanto do imazaquin $\left(\mathrm{pK}_{\mathrm{a}}=3,8\right)$ encontramse na forma molecular, acarretando maior sorção do herbicida por pontes de hidrogênio e interações hidrofóbicas pela matéria orgânica do solo. Regitano et al. (1997, 2001), Oliveira (1998) e Ferreira et al. (2002) também verificaram resultados similares para a sorção do imazaquin em solos brasileiros.

Os coeficientes da equação de regressão linear simples em relação aos valores de $\mathrm{K}_{\mathrm{d}}$ em função dos teores de $\mathrm{MO}$ e $\mathrm{pH}$, separadamente, foram significativos a $5 \%$ de probabilidade e o $\mathrm{R}^{2}$ foi alto para ambas as equações de regressão (Figura 3).

A equação de regressão múltipla ajustada para predizer os valores de $\mathrm{K}_{\mathrm{d}}$ para a população de amostras do solo em função dos 12 valores de matéria orgânica e pH se encontra na Figura 4. Apesar de os termos quadráticos da equação terem sido estatisticamente significativos, não foram incluídos no modelo porque os valores de $\mathrm{pH}$ e matéria orgânica obtidos estão fora dos intervalos amostrados, especialmente em relação aos menores valores de $\mathrm{MO}$ e altos valores de $\mathrm{pH}$. O aumento no teor de matéria orgânica acarretou aumentos nos valores de $\mathrm{K}_{\mathrm{d}}$ nas amostras com baixos valores de $\mathrm{pH}$, sendo estes aumentos mais acentuados naquelas com os menores valores de $\mathrm{pH}$. Em altos valores de $\mathrm{pH}$, o aumento do teor de matéria orgânica acarretou ligeira redução nos valores de $\mathrm{K}_{\mathrm{d}}$ (Figura 4). $\mathrm{O}$ aumento nos valores de $\mathrm{pH}$ acarretou redução nos valores de $\mathrm{K}_{\mathrm{d}}$ em todos os níveis de matéria orgânica. Esta redução foi mais acentuada nos maiores valores de MO. Nas amostras com baixos valores de MO (em torno de

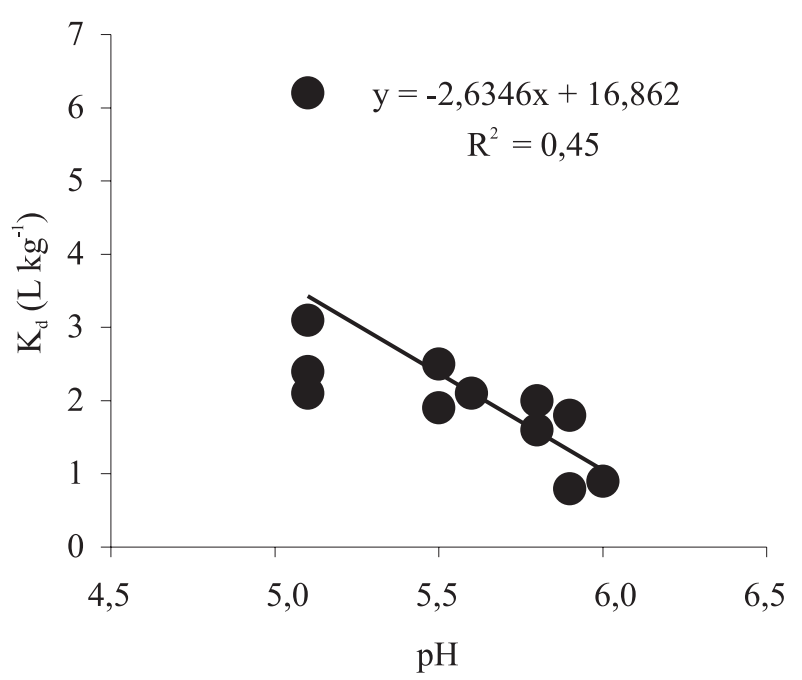

Figura 3. Equações de regressão linear ajustadas para os 12 valores de $\mathrm{K}_{\mathrm{d}}$ em função dos teores de matéria orgânica e pH. 
$2 \%$ ), aumentos nos valores de $\mathrm{pH}$ acarretaram ligeiros aumentos nos valores de $\mathrm{K}_{\mathrm{d}}$.

Goetz et al. (1986) e Rocha et al. (2002) concluíram que os óxidos de ferro e alumínio (hematita e gibbsita) e a caulinita foram os principais constituintes do solo responsáveis pela sorção do imazaquin quando o teor de carbono orgânico foi inferior a 1\%. O modelo de sorção descrito acima mostra que o efeito da matéria orgânica na retenção do herbicida é altamente dependente do $\mathrm{pH}$ do solo, que afeta tanto a natureza iônica da matéria orgânica quanto a especiação do imazaquin. A matéria orgânica possui, entre outros, grupos carboxílicos e fenólicos que apresentam cargas dependentes do $\mathrm{pH}$ com $\mathrm{pK}_{\mathrm{a}}$ de, aproximadamente, 5,2. Em altos valores de
$\mathrm{pH}$, tanto esses grupos funcionais, apresentam-se com carga residual negativa quanto o imazaquin $\left(\mathrm{pH}>\mathrm{pK}_{\mathrm{a}}\right)$, acarretando menor retenção do produto por causa da repulsão das cargas negativas de ambos.

Em solos com baixos valores de matéria orgânica, espera-se que a retenção do imazaquin seja controlada pela carga provinda das argilas, visto que a fração mineral não se apresenta coberta pelo húmus, mas sim por minerais acessórios que podem contribuir com a sorção de moléculas ionogênicas orgânicas (Regitano et al., 1997, 2001). Como o $\mathrm{pH}$ no ponto de carga zero $\left(\mathrm{pH}_{\mathrm{PCZ}}\right)$ desses minerais varia de 6 a 8,5 (Parks \& De Bruyn, 1962), nos valores de $\mathrm{pH}$ abaixo do $\mathrm{pH}_{\mathrm{PCZ}}$, esses minerais apresentam-se como cátions, tendo grande influên-

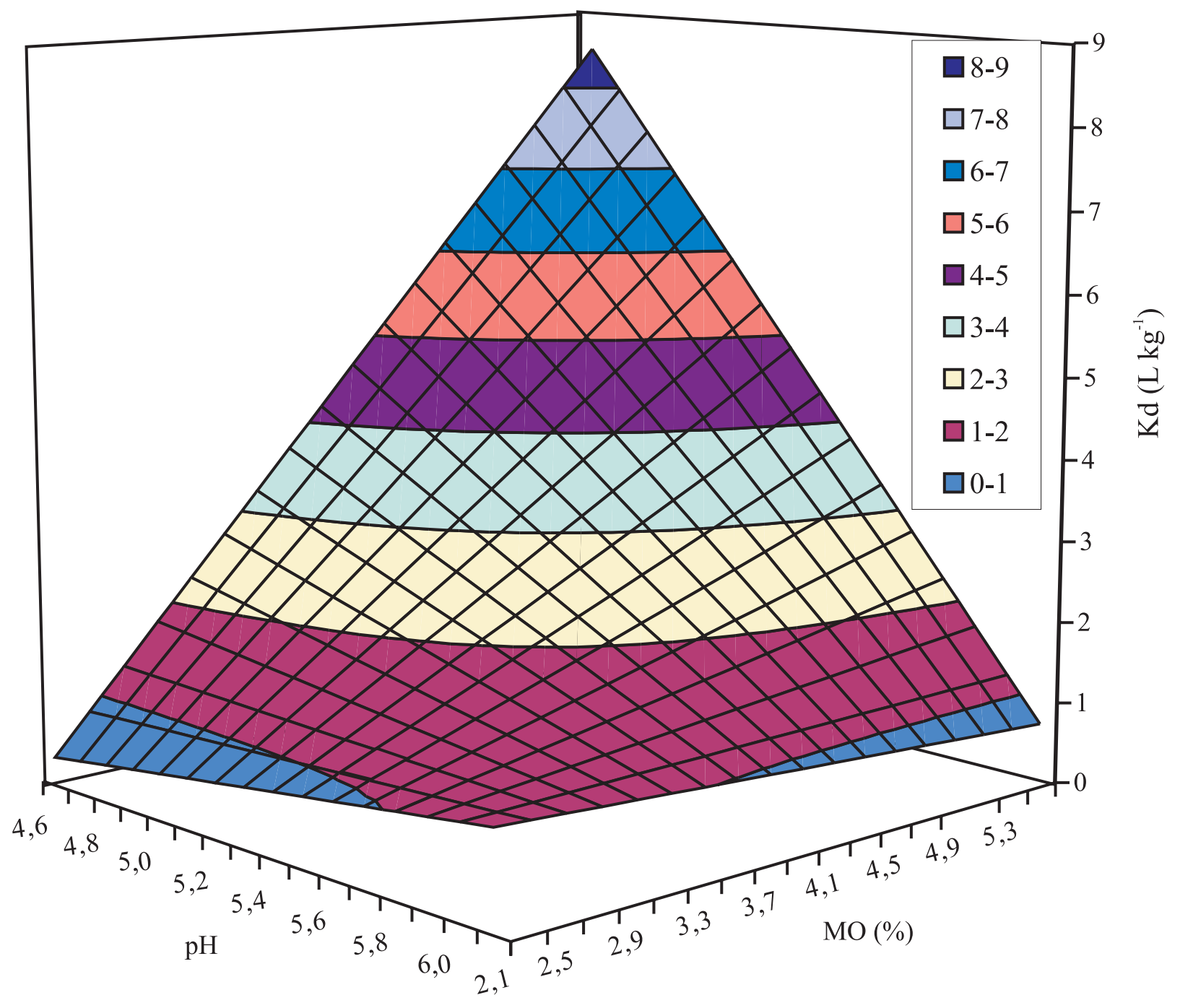

Figura 4. Valores de $\mathrm{K}_{\mathrm{d}}\left(\mathrm{L} \mathrm{kg}^{-1}\right)$ para a retenção do imazaquin no Latossolo Vermelho textura argilosa com diferentes valores de $\mathrm{pH}$ e matéria orgânica. $\mathrm{K}_{\mathrm{d}}=\mathrm{X}_{0}+10,2 \mathrm{MO}+4,11 \mathrm{pH}-1,69 \mathrm{MO} \mathrm{pH} ; \mathrm{R}^{2}=0,91$. 
cia na adsorção de herbicidas ácidos fracos, como picloran e 2,4,5-T e chlorsulfuron (Shea, 1986), principalmente se o $\mathrm{pH}$ é igual ou superior ao $\mathrm{pK}_{\mathrm{a}}$ do herbicida. No entanto, nas amostras com maiores valores de $\mathrm{pH}$, a grande maioria das moléculas do imazaquin apresentam-se com carga negativa, e portanto, a adsorção pode ocorrer por sítios carregados positivamente na superfície dos minerais de argila (Rocha et al., 2002) (Figura 4).

A equação apresentada na Figura 4 foi ajustada utilizando-se somente os coeficientes que medem quanto do herbicida foi retido pelo solo após 24 horas de equilíbrio, não considerando a quantidade do herbicida que poderia retornar para a solução (dessorção). Oliveira Junior et al. (1999) verificaram efeito de histerese na retenção do imazetapir com valores de $\mathrm{n}=1$ (linearidade para a equação de Freundlich) e concluíram que este herbicida não apresentaria tendência de retornar para a solução do solo. Oliveira (1998) também observou histerese na retenção do imazaquin em estudos de sorção e dessorção do imazaquin no Latossolo Vermelho distrófico, textura argilosa. Logo, maior retenção do imazaquin pelo solo indica menor disponibilidade do herbicida para o controle de plantas daninhas havendo, em princípio, a necessidade de aumentar a dose a ser aplicada em áreas sob plantio direto.

A necessidade de utilização dos dois semivariogramas para a geração do mapa de valores de $\mathrm{K}_{\mathrm{d}}$ deve-se à zona anisotrópica nas direções noroeste-sudeste, demonstrando a diferença na variação espacial em ambas as direções (Figura 5). O semivariograma na direção de 35 graus foi ajustado utilizando-se "nugget plus" com estrutura esférica, enquanto o variograma na direção

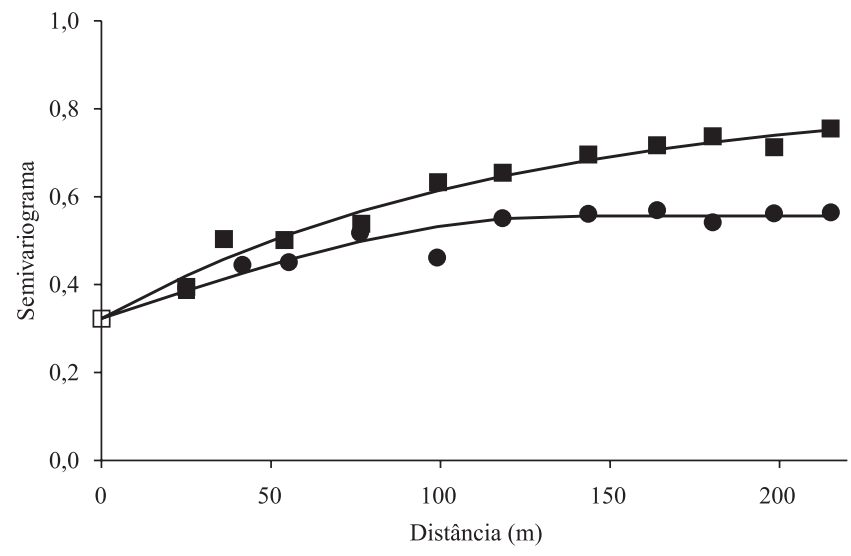

Figura 5. Semivariograma dos valores de matéria orgânica e pH amostrados na direção de $35(\bullet)$ e 125 (ロ) graus na área do pivô central. de 125 graus foi ajustado utilizando o mesmo "nugget plus", porém, com estrutura exponencial. A variável dominante na área foi a matéria orgânica, expressada pelo alto valor de $\mathrm{R}^{2}$ na equação de regressão linear simples e pelo alto coeficiente na equação de regressão múltipla. A grande influência da MO na distribuição espacial do $\mathrm{K}_{\mathrm{d}}$ deve-se à grande variabilidade do $\mathrm{pH}$. A variabilidade relativa do $\mathrm{K}_{\mathrm{d}}(\mathrm{CV}=35 \%)$ foi maior que a obtida para a $\mathrm{MO}(\mathrm{CV}=15 \%)$ ou $\mathrm{pH}(\mathrm{CV}=4 \%)$.

$\mathrm{O}$ mapa gerado com os valores de $\mathrm{K}_{\mathrm{d}}$ mostra que a retenção do imazaquin variou de baixa a moderada (Rocha et al., 2002) para todas as combinações de matéria orgânica e pH (Figura 6). Os maiores valores de $\mathrm{K}_{\mathrm{d}}$ alcançados encontram-se na região noroeste-sudeste, podendo ser atribuídos aos maiores teores de matéria orgânica e aos baixos valores de $\mathrm{pH}$. A área utilizada por longo tempo com manejo convencional, portanto, relativa aos baixos teores de $\mathrm{MO}$, apresentou menores valores de $\mathrm{K}_{\mathrm{d}}$. Admitindo que o herbicida adsorvido pelo solo não está disponível para absorção pelas plantas, a utilização de dose única de imazaquin em áreas de plantio convencional, onde foram observados menores valores de $\mathrm{K}_{\mathrm{d}}$, pode acarretar uma desuniformidade no controle das plantas invasoras, assim como, uma possível lixiviação diferencial do produto, principalmente, quando houver precipitação ou irrigação na área de cultivo.

Oliveira Junior et al. (1999) observaram que a utilização de modelos em escala regional sempre terá alto grau de incerteza em virtude da variabilidade espacial e temporal, necessitando, portanto, de testes e monitoramentos no campo. Os resultados deste trabalho atestaram a importância da variabilidade espacial dos parâmetros que
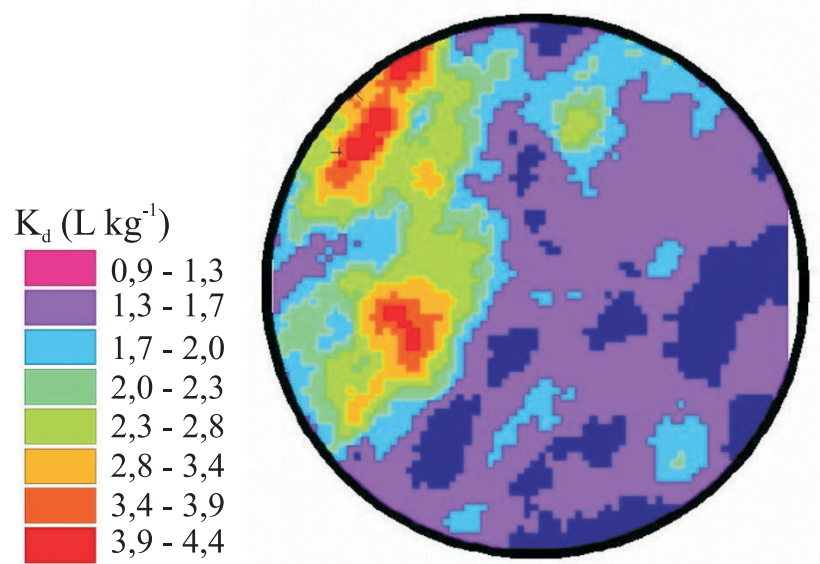

Figura 6. Valores de $\mathrm{K}_{\mathrm{d}}$ obtidos para a população de amostras coletadas na área do Pivô Central. 
afetam a sorção e o destino do imazaquin no solo, que são altamente dependentes das propriedades físico-químicas do herbicida e, principalmente, do conteúdo de matéria orgânica e do pH do solo.

\section{Conclusões}

1. A sorção do imazaquin é variável e maior nas amostras com maiores teores de matéria orgânica e menores valores de $\mathrm{pH}$ do solo.

2. O efeito da matéria orgânica na retenção do imazaquin é dependente do $\mathrm{pH}$.

3. Áreas de Latossolo Vermelho distrófico, textura argilosa, sob plantio direto tendem a apresentar maior retenção do imazaquin do que áreas sob manejo convencional.

\section{Agradecimentos}

Ao Conselho Nacional de Desenvolvimento Científico e Tecnológico, pelo apoio financeiro e à Empresa Brasileira de Pesquisa Agropecuária, pela concessão das instalações e equipamentos.

\section{Referências}

BLUMHORST, M.R.; WEBER, J.B.; SWAIN, L.R. Efficacy of selected herbicides as influenced by soil properties. Weed Technology, v.4, p.279-283, 1990.

CHE, M.; LOUX, M.M.; TRAINA, S.J.; LOGAN, T.J. Effects of $\mathrm{pH}$ on sorption and desorption of imazaquin and imazethapyr on clays and humic acid. Journal of Environmental Quality, v.21, p.698-703, 1992.

DEUTSCH, C.V.; JOURNEL, A.G. GSLIB: Geostatistical Software Library and User's Guide. New York: Oxford University Press, 1997. 340p.

FERREIRA, J.A.; MARTIN-NETO, L.; VAZ, C.M.P.; REGITANO, J.B. Sorption interactions between imazaquin and a humic acid extracted from a typical Brazilian Oxisol. Journal of Environmental Quality, v.31, p.1665-1670, 2002.

GERSTL, Z. An update on the $\mathrm{K}_{\mathrm{oc}}$ concept in regard to regional scale management. Crop Protection, v.19, p.643-648, 2000.

GOETZ, A.J.; WEHTJE, G.; WALKER, R.H.; HAJEK, B. Soil solution and mobility characterization of imazaquin. Weed Science, v.34, p.788-793, 1986.

GONESE, J.U.; WEBER, J.B. Herbicide rate recommendations: soil parameter equations vs. registered rate recommendations. Weed Technology, v.12, p.235-242, 1998.
GOOVAERTS, P. Geostatistics for natural resources evaluation. New York: Oxford University Press, 1997. 496p.

GREEN, R.E.; KARICKHOFF, S.W. Sorption estimates for modeling. In: CHENG, H.H. (Ed.). Pesticides in the soil environment: processes, impacts, and modeling. Madison: Soil Science Society of America, 1990. p.79-101.

ISAAKS, E.H.; SRIVASTAVA, R.M. An introduction to applied geostatistics. New York: Oxford University Press, 1990. 561p.

KOSKINEN, W.C.; HARPER, S.S. The retention process: mechanisms. In: CHENG, H.H. (Ed.). Pesticides in the soil environment: processes, impacts, and modeling. Madison: Soil Science Society of America, 1990. p.51-77.

LOUX, M.M.; LIEBL, R.A.; SLIFE, F.W. Adsorption of imazaquin and imazethapyr on soils, sediments, and selected adsorbents. Weed Science, v.37, p.712-718, 1989.

LOUX, M.M.; REESE, K.D. Effect of soil $\mathrm{pH}$ on adsorption and persistence of imazaquin. Weed Science, v.40, p.490-496, 1992.

MANGELS, G. Behavior of the imidazolinone herbicides in soil: A review of the literature. In: SHANER, D.L.; O'CONNOR, S.L. (Ed.). The imidazolinone herbicides. Boca Raton: CRC Press, 1991. p.191-209.

OLIVEIRA, M.F. de. Retenção dos herbicidas Flazasulfuron e Imazaquin em solos de diferentes classes e hidrólise do Flazasulfuron em diferentes valores de pH e temperatura. 1998. 71p. Tese (Doutorado) - Universidade Estadual do Norte Fluminense, Campos dos Goytacazes.

OLIVEIRA JUNIOR, R.S.; KOSKINEN, W.C.; FERREIRA, F.A.; KHAKURAL, B.R.; MULLA, D.J.; ROBERT, P.J. Spatial variability of imazethapyr sorption in soil. Weed Science, v.47, p.243248, 1999.

PARKS, G.A.; De BRUYN, P.L. The zero point of charge of oxides. Journal of Physical Chemistry, v.66, p.967-973, 1962.

REGITANO, J.B.; ALLEONI, L.R.F.; TORNISIELO, V.L. Atributos de solos tropicais e a sorção do imazaquin. Scientia Agricola, v.58, p.801-807, 2001.

REGITANO, J.B.; BISCHOFF, M.; LEE, L.S.; REICHERT, J.M.; TURCO, R.F. Retention of imazaquin in soil. Environmental Toxicology and Chemistry, v.16, p.397-404, 1997.

ROCHA, W.S.D.; REGITANO, J.B.; ALLEONI, L.R.F.; TORNISIELO, V.F. Sorption of imazaquin in soils with positive balance of charges. Chemosphere, v.49, p.263-270, 2002.

SHEA, P.J. Chlorsulfuron dissociation and adsorption on selected adsorbents and soils. Weed Science, v.34, p.474-478, 1986.

STOURGAARD, R.N.; SHEA, P.J.; MARTIN, A.R. Effect of soil type and $\mathrm{pH}$ on adsorption, mobility, and efficacy of imazaquin and imazethapyr. Weed Science, v.38, p.67-73, 1990.

Recebido em 19 de maio de 2003 e aprovado em 11 de maio de 2004 